\title{
Some inequalities for double integrals and applications for cubature formula
}

\author{
Samet Erden \\ Department of Mathematics, \\ Faculty of Science, Bartin University, \\ Bartın-Turkey \\ email: erdensmt@gmail.com
}

\author{
Mehmet Zeki Sarikaya \\ Department of Mathematics, \\ Faculty of Science and Arts, \\ Düzce University, Konuralp Campus, \\ Düzce-Turkey \\ email: sarikayamz@gmail.com
}

\begin{abstract}
We establish two Ostrowski type inequalities for double integrals of second order partial derivable functions which are bounded. Then, we deduce some inequalities of Hermite-Hadamard type for double integrals of functions whose partial derivatives in absolute value are convex on the co-ordinates on rectangle from the plane. Finally, some applications in Numerical Analysis in connection with cubature formula are given.
\end{abstract}

\section{Introduction}

Let $f:[a, b] \rightarrow \mathbb{R}$ be a differentiable mapping on $(a, b)$ whose derivative $f^{\prime}$ : $(a, b) \rightarrow \mathbb{R}$ is bounded on $(a, b)$, i.e., $\left\|f^{\prime}\right\|_{\infty}=\sup _{t \in(a, b)}\left|f^{\prime}(t)\right|<\infty$. Then, the inequality holds:

$$
\left|f(x)-\frac{1}{b-a} \int_{a}^{b} f(t) d t\right| \leq\left[\frac{1}{4}+\frac{\left(x-\frac{a+b}{2}\right)^{2}}{(b-a)^{2}}\right](b-a)\left\|f^{\prime}\right\|_{\infty}
$$

2010 Mathematics Subject Classification: 26D07, 26D15, 41A55

Key words and phrases: Ostrowski inequality, Hermite-Hadamard inequality, coordinated convex mapping, cubature formula 
for all $x \in[a, b][15]$. The constant $\frac{1}{4}$ is the best possible. This inequality is well known in the literature as the Ostrowski inequality.

Let $f: I \subset \mathbb{R} \rightarrow \mathbb{R}$ be a convex mapping defined on the interval I of real numbers and $a, b \in I$, with $a<b$. The following double inequality is well known in the literature as the Hermite-Hadamard inequality [9]:

$$
f\left(\frac{a+b}{2}\right) \leq \frac{1}{b-a} \int_{a}^{b} f(x) d x \leq \frac{f(a)+f(b)}{2} .
$$

This inequality has attracted considerable attention and interest from mathematicians and other researchers as shown by hundreds of papers published in the last decade one can find by making a simple search in the MathSciNet database of the American Mathematical Society. For example, Bakula et al. presented some Hermite-Hadamard type inequalities for $\mathrm{m}$-convex and $(\alpha, \mathrm{m})$ convex functions in [3].

In a recent paper [2], Barnett and Dragomir proved the following Ostrowski type inequality for double integrals:

Theorem 1 Let $\mathrm{f}:[\mathrm{a}, \mathrm{b}] \times[\mathrm{c}, \mathrm{d}] \rightarrow \mathbb{R}$ be continuous on $[\mathrm{a}, \mathrm{b}] \times[\mathrm{c}, \mathrm{d}], \mathrm{f}_{x, y}^{\prime \prime}=\frac{\partial^{2} \mathrm{f}}{\partial x \partial y}$ exists on $(\mathrm{a}, \mathrm{b}) \times(\mathrm{c}, \mathrm{d})$ and is bounded, i.e.,

$$
\left\|f_{x, y}^{\prime \prime}\right\|_{\infty}=\sup _{(x, y) \in(a, b) \times(c, d)}\left|\frac{\partial^{2} f(x, y)}{\partial x \partial y}\right|<\infty .
$$

Then, we have the inequality:

$$
\begin{aligned}
& \mid \int_{a}^{b} \int_{c}^{d} f(s, t) d t d s-(d-c)(b-a) f(x, y) \\
& \quad-\left[(b-a) \int_{c}^{d} f(x, t) d t+(d-c) \int_{a}^{b} f(s, y) d s\right] \mid \\
& \leq\left[\frac{1}{4}(b-a)^{2}+\left(x-\frac{a+b}{2}\right)^{2}\right]\left[\frac{1}{4}(d-c)^{2}+\left(y-\frac{d+c}{2}\right)^{2}\right]\left\|f_{x, y}^{\prime \prime}\right\|_{\infty}
\end{aligned}
$$

for all $(x, y) \in[a, b] \times[c, d]$.

In [2], the inequality (2) is established by the use of integral identity involving Peano kernels. In [16], Pachpatte obtained a new inequality in the view of (2) by using elementary analysis. Latif et al. proved some Ostrowski type 
inequalities for functions that are co-ordinated convex in [12]. Sarikaya gave integral inequalities for bounded functions in [20]. Authors deduced weighted version of Ostrowski type inequalities for double integrals involving functions of two independent variables by using fairly elementary analysis in [1], [18], [19] and [24].

Let us now consider a bidimensional interval $\Delta=:[a, b] \times[c, d]$ in $\mathbb{R}^{2}$ with $\mathrm{a}<\mathrm{b}$ and $\mathrm{c}<\mathrm{d}$. A mapping $\mathrm{f}: \Delta \rightarrow \mathbb{R}$ is said to be convex on $\Delta$ if the following inequality:

$$
f(t x+(1-t) z, t y+(1-t) w) \leq t f(x, y)+(1-t) f(z, w)
$$

holds, for all $(x, y),(z, w) \in \Delta$ and $t \in[0,1]$. A function $f: \Delta \rightarrow \mathbb{R}$ is said to be on the co-ordinates on $\Delta$ if the partial mappings $f_{y}:[a, b] \rightarrow \mathbb{R}, f_{y}(u)=$ $f(u, y)$ and $f_{x}:[c, d] \rightarrow \mathbb{R}, f_{x}(v)=f(x, v)$ are convex where defined for all $x \in[a, b]$ and $y \in[c, d]$ (see, [5]).

A formal definition for co-ordinated convex function may be stated as follows:

Definition 1 A function $f: \Delta \rightarrow \mathbb{R}$ will be called co-ordinated convex on $\Delta$, for all $\mathrm{t}, \mathrm{s} \in[0,1]$ and $(\mathrm{x}, \mathrm{y}),(\mathrm{u}, \mathrm{v}) \in \Delta$, if the following inequality holds:

$$
\begin{aligned}
& f(t x+(1-t) y, s u+(1-s) v) \\
& \quad \leq t s f(x, u)+s(1-t) f(y, u)+t(1-s) f(x, v)+(1-t)(1-s) f(y, v) .
\end{aligned}
$$

Clearly, every convex function is a co-ordinated convex. Furthermore, there exists co-ordinated convex function which is not convex, (see, [5]).

Also, in [5], Dragomir established the following similar inequality of Hadamard's type for co-ordinated convex mapping on a rectangle from the plane $\mathbb{R}^{2}$.

Theorem 2 Suppose that $f: \Delta \rightarrow \mathbb{R}$ is co-ordinated convex on $\Delta$. Then one has the inequalities:

$$
\begin{aligned}
f\left(\frac{a+b}{2}, \frac{c+d}{2}\right) \leq & \frac{1}{2}\left[\frac{1}{b-a} \int_{a}^{b} f\left(x, \frac{c+d}{2}\right) d x+\frac{1}{d-c} \int_{c}^{d} f\left(\frac{a+b}{2}, y\right) d y\right] \\
\leq & \frac{1}{(b-a)(d-c)} \int_{a}^{b} \int_{c}^{d} f(x, y) d y d x \\
\leq & \frac{1}{4}\left[\frac{1}{b-a} \int_{a}^{b} f(x, c) d x+\frac{1}{b-a} \int_{a}^{b} f(x, d) d x\right. \\
& \left.+\frac{1}{d-c} \int_{c}^{d} f(a, y) d y+\frac{1}{d-c} \int_{c}^{d} f(b, y) d y\right] \\
\leq & \frac{f(a, c)+f(a, d)+f(b, c)+f(b, d)}{4} .
\end{aligned}
$$


The above inequalities are sharp.

In recent years, researchers have studied some integral inequalities by using variety convex functions on the co-ordinates on a rectangle from the plane $\mathbb{R}^{2}$. For example, authors gave some Hadamard's type inequalities involving Riemann-Liouville fractional integrals for convex and s-convex functions on the co-ordinates in [4] and [21]. in [6], Dragomir et al. worked an Ostrowski type inequality for two dimensional integrals in term of $\mathrm{L}_{p}$-norms. Erden and Sarikaya deduced weighted version of Hermite-Hadamard type inequalities for functions whose partial derivatives in absolute value are convex on the coordinates on rectangle from the plane in [7] and [8]. In [10], [12]-[14], [22] and [23], some integral inequalities for differentiable co-ordinated convex mappings are obtained. In [21], Sarikaya et al. proved some new inequalities that give estimate of the difference between the middle and the most right terms of (3) for differentiable co-ordinated convex functions. In [7], [11] and [17], some Hermite-Hadamard type inequalities are developed for variety co-ordinated convex functions.

In this study, we firstly establish an identity for second order partial derivative functions. Then, two inequalities of Ostrowski type for double integrals is gotten by using this identity. Also, Hermite-Hadamard type inequalities for convex mappings on the co-ordinates on the rectangle from the plane are obtained. Finally, some applications of the Ostrowski type inequality developed in this work for cubature formula are given.

\section{Main results}

We need the following lemma so as to prove our main results.

Lemma 1 Let $\mathrm{f}:[\mathrm{a}, \mathrm{b}] \times[\mathrm{c}, \mathrm{d}] \rightarrow \mathbb{R}$ be an absolutely continuous function such that the partial derivative of order 2 exists for all $(t, s) \in[a, b] \times[c, d]$. Then, for all $(\mathrm{x}, \mathrm{y}) \in[\mathrm{a}, \mathrm{b}] \times[\mathrm{c}, \mathrm{d}]$, we have the equality

$$
\begin{aligned}
& \int_{a}^{b} \int_{c}^{d} P_{h}(x, t) Q_{h}(y, s) f_{t s}(t, s) d s d t \\
& =\int_{a}^{b} \int_{c}^{d} f(t, s) d s d t+m_{h}(x) \int_{c}^{d}[f(b, s)-f(a, s)] d s
\end{aligned}
$$




$$
\begin{aligned}
& +m_{h}(y) \int_{a}^{b}[f(t, d)-f(t, c)] d t-(d-c) \int_{a}^{b} f(t, y) d t-(b-a) \int_{c}^{d} f(x, s) d s \\
& +(b-a)(d-c) f(x, y)+m_{h}(x) m_{h}(y)[f(a, c)-f(a, d)-f(b, c)+f(b, d)] \\
& -(d-c) m_{h}(x)[f(b, y)-f(a, y)]-(b-a) m_{h}(y)[f(x, d)-f(x, c)] \\
& =S_{h}(x, y, s, t)
\end{aligned}
$$

for

$$
\begin{aligned}
& P_{h}(x, t):= \begin{cases}\left(t-a-m_{h}(x)\right), & a \leq t<x \\
\left(t-b-m_{h}(x)\right), & x \leq t \leq b\end{cases} \\
& Q_{h}(y, s):= \begin{cases}\left(s-c-m_{h}(y)\right), & c \leq s<y \\
\left(s-d-m_{h}(y)\right), & y \leq s \leq d\end{cases}
\end{aligned}
$$

where $\mathrm{m}_{\mathrm{h}}(\mathrm{x})=\mathrm{h}\left(\mathrm{x}-\frac{\mathrm{a}+\mathrm{b}}{2}\right)$ and $\mathrm{m}_{\mathrm{h}}(\mathrm{y})=\mathrm{h}\left(\mathrm{y}-\frac{\mathrm{c}+\mathrm{d}}{2}\right), \mathrm{h} \in[0,2]$.

Proof. By definitions of $P_{h}(x, t)$ and $Q_{h}(y, s)$, we have

$$
\begin{aligned}
& \int_{a}^{b} \int_{c}^{d} P_{h}(x, t) Q_{h}(y, s) f_{t s}(t, s) d s d t \\
& =\int_{a}^{x} \int_{c}^{y}\left[t-a-m_{h}(x)\right]\left[s-c-m_{h}(y)\right] f_{t s}(t, s) d s d t \\
& +\int_{a}^{x} \int_{y}^{d}\left[t-a-m_{h}(x)\right]\left[s-d-m_{h}(y)\right] f_{t s}(t, s) d s d t \\
& +\int_{x}^{b} \int_{c}^{y}\left[t-b-m_{h}(x)\right]\left[s-c-m_{h}(y)\right] f_{t s}(t, s) d s d t \\
& +\int_{b}^{d}\left[t-b-m_{h}(x)\right]\left[s-d-m_{h}(y)\right] f_{t s}(t, s) d s d t .
\end{aligned}
$$

Now, we examine the above integrals. Applying integration by parts twice for 
the first integral in the right hand side of (5), we find that

$$
\begin{aligned}
& \int_{a}^{x} \int_{c}^{y}\left[t-a-m_{h}(x)\right]\left[s-c-m_{h}(y)\right] f_{t s}(t, s) d s d t \\
& =\left[x-a-m_{h}(x)\right]\left[y-c-m_{h}(y)\right] f(x, y)+\left[y-c-m_{h}(y)\right] m_{h}(x) f(a, y) \\
& \quad-\left[y-c-m_{h}(y)\right] \int_{a}^{x} f(t, y) d t+m_{h}(y)\left[x-a-m_{h}(x)\right] f(x, c) \\
& \quad+m_{h}(x) m_{h}(y) f(a, c)-m_{h}(y) \int_{a}^{x} f(t, c) d t-\left[x-a-m_{h}(x)\right] \int_{c}^{y} f(x, s) d s \\
& \quad-m_{h}(x) \int_{c}^{y} f(a, s) d s+\int_{a}^{x} \int_{c}^{y} f(t, s) d s d t .
\end{aligned}
$$

If we calculate the other integrals in a similar way and then we substitute the results in (5), we obtain desired equality (4) which completes the proof.

Now, we establish a new integral inequality for double integrals and also give some results related to this theorem.

Theorem 3 Suppose that all the assumptions of Lemma 1 hold. If $\mathrm{f}_{\mathrm{ts}}=\frac{\partial^{2} \mathrm{f}}{\partial \mathrm{t} \partial \mathrm{s}}$ exists on $(\mathrm{a}, \mathrm{b}) \times(\mathrm{c}, \mathrm{d})$ and is bounded, i.e.,

$$
\left\|f_{t s}\right\|_{\infty}=\sup _{(t, s) \in(a, b) \times(c, d)}\left|\frac{\partial^{2} f(t, s)}{\partial t \partial s}\right|<\infty .
$$

Then, we have the inequality:

$$
\begin{aligned}
& \left|S_{h}(x, y, s, t)\right| \\
& \leq\left[\left(\frac{b-a}{2}\right)^{2}+\left(x-\frac{a+b}{2}\right)^{2}+(h-2)\left(x-\frac{a+b}{2}\right) m_{h}(x)\right] \\
& \quad \times\left[\left(\frac{d-c}{2}\right)^{2}+\left(y-\frac{c+d}{2}\right)^{2}+(h-2)\left(y-\frac{c+d}{2}\right) m_{h}(y)\right]\left\|f_{t s}\right\|_{\infty}
\end{aligned}
$$

for all $(x, y) \in[a, b] \times[c, d]$, where $m_{h}(x)=h\left(x-\frac{a+b}{2}\right)$ and $m_{h}(y)=$ $h\left(y-\frac{c+d}{2}\right), h \in[0,2]$. 
Proof. Taking absolute value of (4) and using bounded of the mapping $f_{t s}$, we find that

$$
\begin{aligned}
\left|S_{h}(x, y, s, t)\right| \leq & \left\|f_{t s}\right\|_{\infty} \int_{a}^{b} \int_{c}^{d}\left|P_{h}(x, t)\right|\left|Q_{h}(y, s)\right| d s d t \\
= & \left\|f_{t s}\right\|_{\infty}\left[\int_{a}^{x}\left|t-a-m_{h}(x)\right| d t+\int_{x}^{b}\left|t-b-m_{h}(x)\right| d t\right] \\
& \times\left[\int_{c}^{y}\left|s-c-m_{h}(y)\right| d t+\int_{y}^{d}\left|s-d-m_{h}(y)\right| d s\right] .
\end{aligned}
$$

We shall observe the above integrals for the cases $a \leq x \leq \frac{a+b}{2}$ and $\frac{a+b}{2} \leq$ $x \leq b$

For all $a \leq x \leq \frac{a+b}{2}$, we have

$$
\int_{a}^{x}\left|t-a-m_{h}(x)\right| d t=\frac{(x-a)^{2}}{2}-(x-a) m_{h}(x)
$$

and

$$
\int_{x}^{b}\left|t-b-m_{h}(x)\right| d t=\frac{(b-x)^{2}}{2}+(b-x) m_{h}(x)+\left[m_{h}(x)\right]^{2} .
$$

For all $\frac{a+b}{2} \leq x \leq b$, we write

$$
\int_{a}^{x}\left|t-a-m_{h}(x)\right| d t=\frac{(x-a)^{2}}{2}-(x-a) m_{h}(x)+\left[m_{h}(x)\right]^{2}
$$

and

$$
\int_{x}^{b}\left|t-b-m_{h}(x)\right| d t=\frac{(b-x)^{2}}{2}+(b-x) m_{h}(x) .
$$

Then, we get

$$
\begin{aligned}
& \int_{a}^{x}\left|t-a-m_{h}(x)\right| d t+\int_{x}^{b}\left|t-b-m_{h}(x)\right| d t \\
& \quad=\frac{(b-x)^{2}+(x-a)^{2}}{2}+2\left(\frac{a+b}{2}-x\right) m_{h}(x)+\left[m_{h}(x)\right]^{2} .
\end{aligned}
$$


Similarly, we obtain

$$
\begin{aligned}
& \int_{c}^{y}\left|s-c-m_{h}(y)\right| d t+\int_{y}^{d}\left|s-d-m_{h}(y)\right| d s \\
& \quad=\frac{(d-y)^{2}+(y-c)^{2}}{2}+2\left(\frac{c+d}{2}-y\right) m_{h}(y)+\left[m_{h}(y)\right]^{2} .
\end{aligned}
$$

If we substitute the equality (8) and (9) in (7), we easily deduce required inequality (6) which completes the proof.

Remark 1 If we take $\mathrm{x}=\frac{\mathrm{a}+\mathrm{b}}{2}$ and $\mathrm{y}=\frac{\mathrm{c}+\mathrm{d}}{2}$ in Theorem 3, then we have the mid-point inequality

$$
\begin{aligned}
& \mid \int_{a}^{b} \int_{c}^{d} f(t, s) d s d t+(b-a)(d-c) f\left(\frac{a+b}{2}, \frac{c+d}{2}\right) \\
& \quad-(d-c) \int_{a}^{b} f\left(t, \frac{c+d}{2}\right) d t-(b-a) \int_{c}^{d} f\left(\frac{a+b}{2}, s\right) d s \mid \\
& \quad \leq \frac{1}{16}(b-a)^{2}(d-c)^{2}\left\|f_{t s}\right\|_{\infty}
\end{aligned}
$$

which was given by Barnett and Dragomir in [2].

Remark 2 Under the same assumptions of Theorem 3 with $\mathrm{h}=1$ and $(\mathrm{x}, \mathrm{y})=$ $(\mathrm{a}, \mathrm{c})$, then the following inequality holds:

$$
\begin{aligned}
& \mid \frac{f(a, c)+f(a, d)+f(b, c)+f(b, d)}{4}+\frac{1}{(b-a)(d-c)} \int_{a}^{b} \int_{c}^{d} f(t, s) d s d t \\
& \quad-\frac{1}{2}\left[\frac{1}{d-c} \int_{c}^{d}[f(b, s)+f(a, s)] d s+\frac{1}{b-a} \int_{a}^{b}[f(t, d)+f(t, c)] d t\right] \\
& \leq \frac{(b-a)(d-c)}{16}\left\|f_{t s}\right\|_{\infty} .
\end{aligned}
$$

Similarly, if we choose $(x, y)=(a, d)$ or $(x, y)=(b, c)$ or $(x, y)=(b, d)$ for $h=1$ in Theorem 3 , then we deduce inequalities which are the same as the above result. 
Remark 3 If we choose $\mathrm{h}=0$ in Theorem 3, then the inequality (6) reduce to (2).

Theorem 4 Suppose that all the assumptions of Lemma 1 hold. If $\mathrm{f}_{\mathrm{ts}} \in$ $\mathrm{L}_{\mathrm{p}}(\Delta), \frac{1}{\mathrm{p}}+\frac{1}{\mathrm{q}}=1$ and $\mathrm{q}>1$, then we have the inequality

$$
\begin{aligned}
\left|S_{h}(x, y, s, t)\right| \leq & {\left[\frac{\left[b-x+m_{h}(x)\right]^{q+1}+\left[x-a-m_{h}(x)\right]^{q+1}}{q+1}\right]^{\frac{1}{q}} } \\
& {\left[\frac{\left[d-y+m_{h}(y)\right]^{q+1}+\left[y-c-m_{h}(y)\right]^{q+1}}{q+1}\right]^{\frac{1}{q}}\left\|f_{t s}\right\|_{p} }
\end{aligned}
$$

for all $(x, y) \in[a, b] \times[c, d]$, where $\mathrm{m}_{\mathrm{h}}(\mathrm{x})=\mathrm{h}\left(\mathrm{x}-\frac{\mathrm{a}+\mathrm{b}}{2}\right)$ and $\mathrm{m}_{\mathrm{h}}(\mathrm{y})=$ $\mathrm{h}\left(\mathrm{y}-\frac{\mathrm{c}+\mathrm{d}}{2}\right), \mathrm{h} \in[0,2]$. Also, $\left\|\mathrm{f}_{\mathrm{ts}}\right\|_{\mathrm{p}}$ is defined by

$$
\left\|f_{t s}\right\|_{p}=\left(\int_{a}^{b} \int_{c}^{d}\left|\frac{\partial^{2} f(t, s)}{\partial t \partial s}\right|^{p} d s d t\right)^{\frac{1}{p}} .
$$

Proof. Taking absolute value of (4) and using Hölder's inequality, we find that

$$
\begin{aligned}
\left|S_{h}(x, y, s, t)\right| & \leq\left(\int_{a}^{b} \int_{c}^{d}\left|P_{h}(x, t)\right|^{q}\left|Q_{h}(y, s)\right|^{q} d s d t\right)^{\frac{1}{q}}\left(\iint_{a}^{b}\left|\frac{\partial^{2} f(t, s)}{\partial t \partial s}\right|^{p} d s d t\right)^{\frac{1}{p}} \\
= & {\left[\int_{a}^{x}\left|t-a-m_{h}(x)\right|^{q} d t+\int_{x}^{b}\left|t-b-m_{h}(x)\right|^{q} d t\right]^{\frac{1}{q}} } \\
& \times\left[\int_{c}^{y}\left|s-c-m_{h}(y)\right| d t+\int_{y}^{d}\left|s-d-m_{h}(y)\right| d s\right]^{\frac{1}{q}}\left\|f_{t s}\right\|_{p} .
\end{aligned}
$$

We need to examine the above integrals for the cases $a \leq x \leq \frac{a+b}{2}$ and $\frac{\mathrm{a}+\mathrm{b}}{2} \leq \mathrm{x} \leq \mathrm{b}$

For the case of $a \leq x \leq \frac{a+b}{2}$, we get

$$
\int_{a}^{x}\left|t-a-m_{h}(x)\right|^{q} d t=\frac{\left[x-a-m_{h}(x)\right]^{q+1}-\left[-m_{h}(x)\right]^{q+1}}{q+1}
$$


and

$$
\int_{x}^{b}\left|t-b-m_{h}(x)\right|^{q} d t=\frac{\left[b-x+m_{h}(x)\right]^{q+1}+\left[-m_{h}(x)\right]^{q+1}}{q+1} .
$$

For the case of $\frac{a+b}{2} \leq x \leq b$, we obtain

$$
\int_{a}^{x}\left|t-a-m_{h}(x)\right|^{q} d t=\frac{\left[x-a-m_{h}(x)\right]^{q+1}+\left[m_{h}(x)\right]^{q+1}}{q+1}
$$

and

$$
\int_{x}^{b}\left|t-b-m_{h}(x)\right|^{q} d t=\frac{\left[b-x+m_{h}(x)\right]^{q+1}-\left[m_{h}(x)\right]^{q+1}}{q+1} .
$$

Then, we can write

$$
\begin{aligned}
& \int_{a}^{x}\left|t-a-m_{h}(x)\right|^{q} d t+\int_{x}^{b}\left|t-b-m_{h}(x)\right|^{q} d t \\
& =\frac{\left[b-x+m_{h}(x)\right]^{q+1}+\left[x-a-m_{h}(x)\right]^{q+1}}{q+1} .
\end{aligned}
$$

Similarly, we easily deduce the identity

$$
\begin{gathered}
\int_{c}^{y}\left|s-c-m_{h}(y)\right|^{q} d t+\int_{y}^{d}\left|s-d-m_{h}(y)\right|^{q} d s \\
=\frac{\left[d-y+m_{h}(y)\right]^{q+1}+\left[y-c-m_{h}(y)\right]^{q+1}}{q+1} .
\end{gathered}
$$

Using the equality (11) and (12), we easily deduce required inequality (10). Hence, the proof is completed.

Remark 4 If we take $\mathrm{x}=\frac{\mathrm{a}+\mathrm{b}}{2}$ and $\mathrm{y}=\frac{\mathrm{c}+\mathrm{d}}{2}$ in Theorem 4, then we have the mid-point inequality

$$
\mid \int_{a}^{b} \int_{c}^{d} f(t, s) d s d t+(b-a)(d-c) f\left(\frac{a+b}{2}, \frac{c+d}{2}\right)
$$




$$
\begin{aligned}
& -(d-c) \int_{a}^{b} f\left(t, \frac{c+d}{2}\right) d t-(b-a) \int_{c}^{d} f\left(\frac{a+b}{2}, s\right) d s \mid \\
& \leq \frac{(b-a)^{1+\frac{1}{q}}(d-c)^{1+\frac{1}{q}}}{4(q+1)^{\frac{2}{q}}}\left\|f_{t s}\right\|_{p}
\end{aligned}
$$

which was given by Dragomir et al. in [6].

Remark 5 If we choose $\mathrm{h}=0$ in Theorem 4, then we have

$$
\begin{aligned}
& \mid(b-a)(d-c) f(x, y)-(d-c) \int_{a}^{b} f(t, y) d t \\
& -(b-a) \int_{c}^{d} f(x, s) d s+\int_{a}^{b} \int_{c}^{d} f(t, s) d s d t \mid \\
& \leq\left\|\frac{\partial^{n+m} f}{\partial t^{n} \partial s^{m}}\right\|_{p}\left[\frac{(x-a)^{q+1}+(b-x)^{q+1}}{q+1}\right]^{\frac{1}{q}} \\
& \quad \times\left[\frac{(y-c)^{q+1}+(d-y)^{q+1}}{q+1}\right]^{\frac{1}{q}}
\end{aligned}
$$

which was proved by Dragomir et al. in [6].

Remark 6 Under the same assumptions of Theorem 4 with $\mathrm{h}=1$ and $(\mathrm{x}, \mathrm{y})=$ $(\mathrm{a}, \mathrm{c})$, then the following inequality holds:

$$
\begin{aligned}
& \mid \frac{f(a, c)+f(a, d)+f(b, c)+f(b, d)}{4}+\frac{1}{(b-a)(d-c)} \int_{a}^{b} \int_{c}^{d} f(t, s) d s d t \\
& \quad-\frac{1}{2}\left[\frac{1}{d-c} \int_{c}^{d}[f(b, s)+f(a, s)] d s+\frac{1}{b-a} \int_{a}^{b}[f(t, d)+f(t, c)] d t\right] \mid \\
& \leq \frac{(b-a)^{1+\frac{1}{q}}(d-c)^{1+\frac{1}{q}}}{4(q+1)^{\frac{2}{q}}}\left\|f_{t s}\right\|_{\infty} .
\end{aligned}
$$

Similarly, if we choose $(x, y)=(a, d)$ or $(x, y)=(b, c)$ or $(x, y)=(b, d)$ for $\mathrm{h}=1$ in Theorem 4 , then we deduce inequalities which are the same as the above result. 
For convenience, we give the following notations used to simplify the details of the next theorem,

$$
\begin{aligned}
A= & (b-a)\left[\frac{(x-a)^{2}}{2}-(x-a) m_{h}(x)\right]+\frac{(b-x)^{3}-(x-a)^{3}}{3} \\
& +\left[\left(\frac{b-a}{2}\right)^{2}+\left(x-\frac{a+b}{2}\right)^{2}\right] m_{h}(x)-\frac{\left[m_{h}(x)\right]^{3}}{3} \\
B= & (b-a)\left[\frac{(b-x)^{2}}{2}+(b-x) m_{h}(x)\right]-\frac{(b-x)^{3}-(x-a)^{3}}{3} \\
& -\left[\left(\frac{b-a}{2}\right)^{2}+\left(x-\frac{a+b}{2}\right)^{2}\right] m_{h}(x)+\frac{\left[m_{h}(x)\right]^{3}}{3} \\
C= & (d-c)\left[\frac{(y-c)^{2}}{2}-(y-c) m_{h}(y)\right]+\frac{(d-y)^{3}-(y-c)^{3}}{3} \\
& +\left[\left(\frac{d-c}{2}\right)^{2}+\left(y-\frac{c+d}{2}\right)^{2}\right] m_{h}(y)-\frac{\left[m_{h}(y)\right]^{3}}{3}
\end{aligned}
$$

and

$$
\begin{aligned}
D= & (d-c)\left[\frac{(d-y)^{2}}{2}+(d-y) m_{h}(y)\right]-\frac{(d-y)^{3}-(y-c)^{3}}{3} \\
& -\left[\left(\frac{d-c}{2}\right)^{2}+\left(y-\frac{c+d}{2}\right)^{2}\right] m_{h}(y)+\frac{\left[m_{h}(y)\right]^{3}}{3} .
\end{aligned}
$$

We give some inequalities by using convexity of $\left|f_{t s}(t, s)\right|$ in the following theorem.

Theorem 5 Suppose that all the assumptions of Lemma 1 hold. If $\left|\mathbf{f}_{\mathrm{ts}}(\mathbf{t}, \mathbf{s})\right|$ is a convex function on the co-ordinates on $[\mathrm{a}, \mathrm{b}] \times[\mathrm{c}, \mathrm{d}]$, then the following inequalities hold:

$$
\begin{aligned}
& \left|S_{h}(x, y, s, t)\right| \\
& \leq \frac{\left|f_{t s}(a, c)\right|}{(b-a)(d-c)} A C+\frac{\left|f_{t s}(a, d)\right|}{(b-a)(d-c)} A\left[D+(d-c)\left[m_{h}(y)\right]^{2}\right] \\
& \quad+\frac{\left|f_{t s}(b, c)\right|}{(b-a)(d-c)}\left[B+(b-a)\left[m_{h}(x)\right]^{2}\right] C \\
& \quad+\frac{\left|f_{t s}(b, d)\right|}{(b-a)(d-c)}\left[B+(b-a)\left[m_{h}(x)\right]^{2}\right]\left[D+(d-c)\left[m_{h}(y)\right]^{2}\right]
\end{aligned}
$$


for all $\mathrm{a} \leq \mathrm{x} \leq \frac{\mathrm{a}+\mathrm{b}}{2}$ and $\mathrm{c} \leq \mathrm{y} \leq \frac{\mathrm{c}+\mathrm{d}}{2}$

$$
\begin{aligned}
& \left|S_{h}(x, y, s, t)\right| \\
& \leq \leq \frac{\left|f_{t s}(a, c)\right|}{(b-a)(d-c)} A\left[C+(d-c)\left[m_{h}(y)\right]^{2}\right]+\frac{\left|f_{t s}(a, d)\right|}{(b-a)(d-c)} A D \\
& \quad+\frac{\left|f_{t s}(b, c)\right|}{(b-a)(d-c)}\left[B+(b-a)\left[m_{h}(x)\right]^{2}\right]\left[C+(d-c)\left[m_{h}(y)\right]^{2}\right] \\
& \quad+\frac{\left|f_{t s}(b, d)\right|}{(b-a)(d-c)}\left[B+(b-a)\left[m_{h}(x)\right]^{2}\right] D
\end{aligned}
$$

for all $\mathrm{a} \leq \mathrm{x} \leq \frac{\mathrm{a}+\mathrm{b}}{2}$ and $\frac{\mathrm{c}+\mathrm{d}}{2} \leq \mathrm{y} \leq \mathrm{d}$

$$
\begin{aligned}
& \left|S_{h}(x, y, s, t)\right| \\
& \leq \leq \frac{\left|f_{t s}(a, c)\right|}{(b-a)(d-c)}\left[A+(b-a)\left[m_{h}(x)\right]^{2}\right] C \\
& \quad+\frac{\left|f_{t s}(a, d)\right|}{(b-a)(d-c)}\left[A+(b-a)\left[m_{h}(x)\right]^{2}\right]\left[D+(d-c)\left[m_{h}(y)\right]^{2}\right] \\
& \quad+\frac{\left|f_{t s}(b, c)\right|}{(b-a)(d-c)} B C+\frac{\left|f_{t s}(b, d)\right|}{(b-a)(d-c)} B\left[D+(d-c)\left[m_{h}(y)\right]^{2}\right]
\end{aligned}
$$

for all $\frac{\mathrm{a}+\mathrm{b}}{2} \leq \mathrm{x} \leq \mathrm{b}$ and $\mathrm{c} \leq \mathrm{y} \leq \frac{\mathrm{c}+\mathrm{d}}{2}$

$$
\begin{aligned}
& \left|S_{h}(x, y, s, t)\right| \\
& \leq \leq \frac{\left|f_{t s}(a, c)\right|}{(b-a)(d-c)}\left[A+(b-a)\left[m_{h}(x)\right]^{2}\right]\left[C+(d-c)\left[m_{h}(y)\right]^{2}\right] \\
& \quad+\frac{\left|f_{t s}(a, d)\right|}{(b-a)(d-c)}\left[A+(b-a)\left[m_{h}(x)\right]^{2}\right] D \\
& \quad+\frac{\left|f_{t s}(b, c)\right|}{(b-a)(d-c)} B\left[C+(d-c)\left[m_{h}(y)\right]^{2}\right]+\frac{\left|f_{t s}(b, d)\right|}{(b-a)(d-c)} B D
\end{aligned}
$$

for all $\frac{\mathrm{a}+\mathrm{b}}{2} \leq \mathrm{x} \leq \mathrm{b}$ and $\frac{\mathrm{c}+\mathrm{d}}{2} \leq \mathrm{y} \leq \mathrm{d}$, where $\mathrm{m}_{\mathrm{h}}(\mathrm{x})=\mathrm{h}\left(\mathrm{x}-\frac{\mathrm{a}+\mathrm{b}}{2}\right)$ and $\mathrm{m}_{\mathrm{h}}(\mathrm{y})=\mathrm{h}\left(\mathrm{y}-\frac{\mathrm{c}+\mathrm{d}}{2}\right), \mathrm{h} \in[0,2]$.

Proof. If we take absolute value of (4), then we get

$$
\left|S_{h}(x, y, s, t)\right| \leq \int_{a}^{b} \int_{c}^{d}\left|P_{h}(x, t)\right|\left|Q_{h}(y, s)\right|\left|f_{t s}(t, s)\right| d s d t .
$$


Since $\left|f_{t s}(t, s)\right|$ is a convex function on the co-ordinates on $[a, b] \times[c, d]$, we have

$$
\begin{aligned}
& \left|f_{t s}\left(\frac{b-t}{b-a} a+\frac{t-a}{b-a} b, \frac{d-s}{d-c} c+\frac{s-c}{d-c} d\right)\right| \\
& \leq \frac{(b-t)(d-s)}{(b-a)(d-c)}\left|f_{t s}(a, c)\right|+\frac{(b-t)(s-c)}{(b-a)(d-c)}\left|f_{t s}(a, d)\right| \\
& \quad+\frac{(t-a)(d-s)}{(b-a)(d-c)}\left|f_{t s}(b, c)\right|+\frac{(t-a)(s-c)}{(b-a)(d-c)}\left|f_{t s}(b, d)\right| .
\end{aligned}
$$

Utilizing the inequality (17), we obtain

$$
\begin{aligned}
& \left|S_{h}(x, y, s, t)\right| \\
& \leq \frac{\left|f_{t s}(a, c)\right|}{(b-a)(d-c)}\left[\int_{a}^{b}(b-t)\left|P_{h}(x, t)\right| d t\right]\left[\int_{c}^{d}(d-s)\left|Q_{h}(y, s)\right| d s\right] \\
& +\frac{\left|f_{t s}(a, d)\right|}{(b-a)(d-c)}\left[\int_{a}^{b}(b-t)\left|P_{h}(x, t)\right| d t\right]\left[\int_{c}^{d}(s-c)\left|Q_{h}(y, s)\right| d s\right] \\
& +\frac{\left|f_{t s}(b, c)\right|}{(b-a)(d-c)}\left[\int_{a}^{b}(t-a)\left|P_{h}(x, t)\right| d t\right]\left[\int_{c}^{d}(d-s)\left|Q_{h}(y, s)\right| d s\right] \\
& +\frac{\left|f_{t s}(b, d)\right|}{(b-a)(d-c)}\left[\int_{a}^{b}(t-a)\left|P_{h}(x, t)\right| d t\right]\left[\int_{c}^{d}(s-c)\left|Q_{h}(y, s)\right| d s\right] .
\end{aligned}
$$

We observe that

$$
\begin{aligned}
& \int_{a}^{b}(b-t)\left|P_{h}(x, t)\right| d t=(b-a) \int_{a}^{x}\left|t-a-m_{h}(x)\right| d t \\
& \quad-\int_{a}^{x}(t-a)\left|t-a-m_{h}(x)\right| d t+\int_{x}^{b}(b-t)\left|t-b-m_{h}(x)\right| d t .
\end{aligned}
$$

Now, let us observe that

$$
\begin{aligned}
\int_{p}^{r}|t-p||t-q| d t & =\int_{p}^{q}(t-p)(q-t) d t+\int_{q}^{r}(t-p)(t-q) d t \\
& =\frac{(q-p)^{3}}{3}+\frac{(r-p)^{3}}{3}-\frac{(q-p)(r-p)^{2}}{2}
\end{aligned}
$$


for all $r, p, q$ such that $p \leq q \leq r$.

We investigate integrals given in the equality (19) for the cases $a \leq x \leq \frac{a+b}{2}$ and $\frac{a+b}{2} \leq x \leq b$;

For all $a \leq x \leq \frac{a+b}{2}$, we have

$$
\begin{aligned}
& \int_{a}^{x}\left|t-a-m_{h}(x)\right| d t=\frac{(x-a)^{2}}{2}-(x-a) m_{h}(x), \\
& \int_{a}^{x}(t-a)\left|t-a-m_{h}(x)\right| d t=\frac{(x-a)^{3}}{3}-\frac{(x-a)^{2}}{2} m_{h}(x)
\end{aligned}
$$

and using the equality (20) for second integral, we get

$$
\int_{x}^{b}|b-t|\left|t-b-m_{h}(x)\right| d t=-\frac{\left[m_{h}(x)\right]^{3}}{3}+\frac{(b-x)^{3}}{3}+\frac{(b-x)^{2}}{2} m_{h}(x) .
$$

For all $\frac{a+b}{2} \leq x \leq b$, we have

$$
\begin{aligned}
& \int_{a}^{x}\left|t-a-m_{h}(x)\right| d t=\frac{(x-a)^{2}}{2}-(x-a) m_{h}(x)+\left[m_{h}(x)\right]^{2}, \\
& \int_{x}^{b}|b-t|\left|t-b-m_{h}(x)\right| d t=\frac{(b-x)^{3}}{3}+\frac{(b-x)^{2}}{2} m_{h}(x)
\end{aligned}
$$

and using the equality (20), we obtain

$$
\int_{a}^{x}|a-t|\left|t-a-m_{h}(x)\right| d t=\frac{\left[m_{h}(x)\right]^{3}}{3}+\frac{(x-a)^{3}}{3}-\frac{(x-a)^{2}}{2} m_{h}(x) .
$$

Then, we can write

$$
\int_{a}^{b}(b-t)\left|P_{h}(x, t)\right| d t=A
$$

for all $a \leq x \leq \frac{a+b}{2}$ and

$$
\int_{a}^{b}(b-t)\left|P_{h}(x, t)\right| d t=A+(b-a)\left[m_{h}(x)\right]^{2}
$$


for all $\frac{a+b}{2}<x \leq b$.

Similarly, we can easily find the other integrals given in the inequality (18) for cases $\mathrm{a} \leq \mathrm{x} \leq \frac{\mathrm{a}+\mathrm{b}}{2}, \frac{\mathrm{a}+\mathrm{b}}{2}<\mathrm{x} \leq \mathrm{b}, \mathrm{c} \leq \mathrm{y} \leq \frac{\mathrm{c}+\mathrm{d}}{2}$ and $\frac{\mathrm{c}+\mathrm{d}}{2} \leq \mathrm{y} \leq \mathrm{d}$. If we substitute the resulting inequalities for all cases in (18), we obtain desired inequalities. The proof is thus completed.

Remark 7 If we take $\mathrm{x}=\frac{\mathrm{a}+\mathrm{b}}{2}$ and $\mathrm{y}=\frac{\mathrm{c}+\mathrm{d}}{2}$ in Theorem 5, then we have the mid-point inequality

$$
\begin{aligned}
& \mid \int_{a}^{b} \int_{c}^{d} f(t, s) d s d t+(b-a)(d-c) f\left(\frac{a+b}{2}, \frac{c+d}{2}\right) \\
& \quad-(d-c) \int_{a}^{b} f\left(t, \frac{c+d}{2}\right) d t-(b-a) \int_{c}^{d} f\left(\frac{a+b}{2}, s\right) d s \mid \\
& \leq \frac{(b-a)^{2}(d-c)^{2}}{16}\left[\frac{\left|f_{t s}(a, c)\right|+\left|f_{t s}(a, d)\right|+\left|f_{t s}(b, c)\right|+\left|f_{t s}(b, d)\right|}{4}\right]
\end{aligned}
$$

which was given by Latif and Dragomir in [12].

Corollary 1 Under the same assumptions of Theorem 5 with $\mathrm{h}=0$, we get the inequality

$$
\begin{aligned}
& \mid \int_{a}^{b} \int_{c}^{b} f(s, t) d t d s+(d-c)(b-a) f(x, y) \\
& \quad-\left[(b-a) \int_{c}^{d} f(x, t) d t+(d-c) \int_{a}^{b} f(s, y) d s\right] \mid \\
& \leq \quad\left[(b-a) \frac{(x-a)^{2}}{2}+\frac{(b-x)^{3}-(x-a)^{3}}{3}\right] \\
& \quad \times\left\{\frac{\left|f_{t s}(a, c)\right|}{(b-a)(d-c)}\left[(d-c) \frac{(y-c)^{2}}{2}+\frac{(d-y)^{3}-(y-c)^{3}}{3}\right]\right. \\
& \left.\quad+\frac{\left|f_{t s}(a, d)\right|}{(b-a)(d-c)}\left[(d-c) \frac{(d-y)^{2}}{2}-\frac{(d-y)^{3}-(y-c)^{3}}{3}\right]\right\} \\
& \quad+\left[(b-a) \frac{(b-x)^{2}}{2}-\frac{(b-x)^{3}-(x-a)^{3}}{3}\right]
\end{aligned}
$$




$$
\begin{aligned}
& \times\left\{\frac{\left|f_{t s}(b, c)\right|}{(b-a)(d-c)}\left[(d-c) \frac{(y-c)^{2}}{2}+\frac{(d-y)^{3}-(y-c)^{3}}{3}\right]\right. \\
& \left.+\frac{\left|f_{t s}(b, d)\right|}{(b-a)(d-c)}\left[(d-c) \frac{(d-y)^{2}}{2}-\frac{(d-y)^{3}-(y-c)^{3}}{3}\right]\right\}
\end{aligned}
$$

for all $(x, y) \in[a, b] \times[c, d]$.

Remark 8 If we take $(x, y)=(a, c)$ for $\mathrm{h}=1$ in the inequality (13), then we have the result

$$
\begin{aligned}
& \mid \frac{f(a, c)+f(a, d)+f(b, c)+f(b, d)}{4}+\frac{1}{(b-a)(d-c)} \int_{a}^{b} \int_{c}^{d} f(t, s) d s d t \\
& \quad-\frac{1}{2}\left[\frac{1}{d-c} \int_{c}^{d}[f(b, s)+f(a, s)] d s+\frac{1}{b-a} \int_{a}^{b}[f(t, d)+f(t, c)] d t\right] \mid \\
& \leq \frac{(b-a)(d-c)}{16}\left[\frac{\left|f_{t s}(a, c)\right|+\left|f_{t s}(a, d)\right|+\left|f_{t s}(b, c)\right|+\left|f_{t s}(b, d)\right|}{4}\right]
\end{aligned}
$$

which was proved Sarikaya et al. in [21].

Similarly, if we choose $(x, y)=(a, d)$ in $(14)$ or $(x, y)=(b, c)$ in $(15)$ or $(x, y)=(b, d)$ in $(16)$ for $h=1$, then we obtain inequalities which are the same as the above result.

Theorem 6 Suppose that all the assumptions of Lemma 1 hold. If $\left|\mathrm{f}_{\mathrm{ts}}(\mathrm{t}, \mathrm{s})\right|^{\mathrm{q}}$ is a convex function on the co-ordinates on $[\mathrm{a}, \mathrm{b}] \times[\mathrm{c}, \mathrm{d}], \frac{1}{\mathrm{p}}+\frac{1}{\mathrm{q}}=1$ and $\mathrm{q}>1$, then the following inequality holds:

$$
\begin{aligned}
& \left|S_{h}(x, y, s, t)\right| \\
& \leq(b-a)^{\frac{1}{q}}(d-c)^{\frac{1}{q}}\left[\frac{\left[b-x+m_{h}(x)\right]^{p+1}+\left[x-a-m_{h}(x)\right]^{p+1}}{p+1}\right]^{\frac{1}{p}} \\
& \quad \times\left[\frac{\left[d-y+m_{h}(y)\right]^{p+1}+\left[y-c-m_{h}(y)\right]^{p+1}}{p+1}\right]^{\frac{1}{p}} \\
& \quad \times\left\{\frac{\left|f_{t s}(a, c)\right|^{q}+\left|f_{t s}(a, d)\right|^{q}+\left|f_{t s}(b, c)\right|^{q}+\left|f_{t s}(b, d)\right|^{q}}{4}\right\}^{\frac{1}{q}}
\end{aligned}
$$

for all $(x, y) \in[a, b] \times[c, d]$, where $m_{h}(x)=h\left(x-\frac{a+b}{2}\right)$ and $m_{h}(y)=$ $h\left(y-\frac{c+d}{2}\right), h \in[0,2]$. 
Proof. Taking absolute value of (4) and using Hölder's inequality, we find that $\left|S_{h}(x, y, s, t)\right| \leq\left(\int_{a}^{b} \int_{c}^{d}\left|P_{h}(x, t)\right|^{p}\left|Q_{h}(y, s)\right|^{p} d s d t\right)^{\frac{1}{q}}\left(\iint_{a}^{b}\left|f_{t s}^{d}(t, s)\right|^{q} d s d t\right)^{\frac{1}{q}}$.

By similar methods in the proof of Theorem 4, we obtain

$$
\begin{aligned}
& {\left[\int_{a}^{b} \int_{c}^{d}\left|P_{h}(x, t)\right|^{p}\left|Q_{h}(y, s)\right|^{p} d s d t\right]^{\frac{1}{q}}} \\
& =\left[\frac{\left[b-x+m_{h}(x)\right]^{p+1}+\left[x-a-m_{h}(x)\right]^{p+1}}{p+1}\right]^{\frac{1}{p}} \\
& \quad \times\left[\frac{\left[d-y+m_{h}(y)\right]^{p+1}+\left[y-c-m_{h}(y)\right]^{p+1}}{p+1}\right]^{\frac{1}{p}} .
\end{aligned}
$$

Since $\left|f_{t s}(t, s)\right|^{q}$ is a convex function on the co-ordinates on $\Delta$, we have

$$
\begin{aligned}
\mid f_{t s} & \left.\left(\frac{b-t}{b-a} a+\frac{t-a}{b-a} b, \frac{d-s}{d-c} c+\frac{s-c}{d-c} d\right)\right|^{q} \\
\leq & \frac{(b-t)(d-s)}{(b-a)(d-c)}\left|f_{t s}(a, c)\right|^{q}+\frac{(b-t)(s-c)}{(b-a)(d-c)}\left|f_{t s}(a, d)\right|^{q} \\
& \quad+\frac{(t-a)(d-s)}{(b-a)(d-c)}\left|f_{t s}(b, c)\right|^{q}+\frac{(t-a)(s-c)}{(b-a)(d-c)}\left|f_{t s}(b, d)\right|^{q} .
\end{aligned}
$$

Using the inequality (22), it follows that

$$
\begin{aligned}
& \left(\int_{a}^{b} \int_{c}^{d}\left|f_{t s}(t, s)\right|^{q} d s d t\right)^{\frac{1}{q}} \leq(b-a)^{\frac{1}{q}}(d-c)^{\frac{1}{q}} \\
& \quad \times\left\{\frac{\left|f_{t s}(a, c)\right|^{q}+\left|f_{t s}(a, d)\right|^{q}+\left|f_{t s}(b, c)\right|^{q}+\left|f_{t s}(b, d)\right|^{q}}{4}\right\}^{\frac{1}{q}} .
\end{aligned}
$$

The proof is thus completed.

Remark 9 If we take $\mathrm{x}=\frac{\mathrm{a}+\mathrm{b}}{2}$ and $\mathrm{y}=\frac{\mathrm{c}+\mathrm{d}}{2}$ in Theorem 6, then we have the mid-point inequality

$$
\mid \int_{a}^{b} \int_{c}^{d} f(t, s) d s d t+(b-a)(d-c) f\left(\frac{a+b}{2}, \frac{c+d}{2}\right)
$$




$$
\begin{aligned}
& -(d-c) \int_{a}^{b} f\left(t, \frac{c+d}{2}\right) d t-(b-a) \int_{c}^{d} f\left(\frac{a+b}{2}, s\right) d s \mid \\
\leq & \frac{(b-a)^{2}(d-c)^{2}}{4(q+1)^{\frac{2}{q}}}\left\{\frac{\left|f_{t s}(a, c)\right|^{q}+\left|f_{t s}(a, d)\right|^{q}+\left|f_{t s}(b, c)\right|^{q}+\left|f_{t s}(b, d)\right|^{q}}{4}\right\}^{\frac{1}{q}}
\end{aligned}
$$

which was deduced by Latif and Dragomir in [12].

Corollary 2 If we choose $\mathrm{h}=0$ in Theorem 6 , then we have

$$
\begin{aligned}
& \mid(b-a)(d-c) f(x, y)-(d-c) \int_{a}^{b} f(t, y) d t \\
& \quad-(b-a) \int_{c}^{d} f(x, s) d s+\int_{a} \int_{c} f(t, s) d s d t \mid \\
& \leq(b-a)^{\frac{1}{q}}\left[\frac{(b-x)^{p+1}+(x-a)^{p+1}}{p+1}\right]^{\frac{1}{p}} \\
& \quad \times(d-c)^{\frac{1}{q}}\left[\frac{(d-y)^{p+1}+(y-c)^{p+1}}{p+1}\right]^{\frac{1}{p}} \\
& \quad \times\left\{\frac{\left|f_{t s}(a, c)\right|^{q}+\left|f_{t s}(a, d)\right|^{q}+\left|f_{t s}(b, c)\right|^{q}+\left|f_{t s}(b, d)\right|^{q}}{4}\right\}^{\frac{1}{q}}
\end{aligned}
$$

which is a Ostrowski type inequality for co-ordinated convex mappings.

Remark 10 Under the same assumptions of Theorem 6 with $\mathrm{h}=1$ and $(\mathrm{x}, \mathrm{y})=(\mathrm{a}, \mathrm{c})$, then the following inequality holds:

$$
\begin{aligned}
& \mid \frac{f(a, c)+f(a, d)+f(b, c)+f(b, d)}{4}+\frac{1}{(b-a)(d-c)} \int_{a}^{b} \int_{c}^{d} f(t, s) d s d t \\
& \quad-\frac{1}{2}\left[\frac{1}{d-c} \int_{c}^{d}[f(b, s)+f(a, s)] d s+\frac{1}{b-a} \int_{a}^{b}[f(t, d)+f(t, c)] d t\right] \mid \\
& \leq \frac{(b-a)^{2}(d-c)^{2}}{4(q+1)^{\frac{2}{q}}}\left\{\frac{\left|f_{t s}(a, c)\right|^{q}+\left|f_{t s}(a, d)\right|^{q}+\left|f_{t s}(b, c)\right|^{q}+\left|f_{t s}(b, d)\right|^{q}}{4}\right\}^{\frac{1}{q}}
\end{aligned}
$$

which was proved Sarikaya et al. in [21]. 
Similarly, if we choose $(x, y)=(a, d)$ or $(x, y)=(b, c)$ or $(x, y)=(b, d)$ for $\mathrm{h}=1$ in Theorem 6 , then we deduce inequalities which are the same as the above result.

\section{Applications to cubature formulae}

We now consider applications of the integral inequalities developed in the previous section, to obtain estimates of cubature formula which, it turns out have a markedly smaller error than that which may be obtained by the classical results.

Let $I_{n}: a=x_{0}<x_{1}<\ldots<x_{n-1}<x_{n}=b$ and $J_{m}: c=y_{0}<y_{1}<\ldots<$ $y_{m-1}<y_{m}=d$ be divisions of the intervals $[a, b]$ and $[c, d], \xi_{i} \in\left[x_{i}, x_{i+1}\right]$ $(i=0, \ldots, n-1)$ and $\eta_{j} \in\left[y_{j}, y_{j+1}\right](j=0, \ldots, m-1)$. Consider the sum

$$
\begin{aligned}
T\left(f, I_{n}, J_{m}, \xi, \eta\right):= & \sum_{i=0}^{n-1} \sum_{j=0}^{m-1} l_{j} \int_{x_{i}}^{x_{i+1}} f\left(t, \eta_{j}\right) d t \\
& +\sum_{i=0}^{n-1} \sum_{j=0}^{m-1} k_{i} \int_{y_{j}}^{y_{j+1}} f\left(\xi_{i}, s\right) d s-\sum_{i=0}^{n-1} \sum_{j=0}^{m-1} k_{i} l_{j} f\left(\xi_{i}, \eta_{j}\right) \\
& -\sum_{i=0}^{n-1} \sum_{j=0}^{m-1} m_{h}\left(\xi_{i}\right) \int_{y_{j}}^{y_{j+1}}\left[f\left(x_{i+1}, s\right)-f\left(x_{i}, s\right)\right] d s \\
& -\sum_{i=0}^{n-1} \sum_{j=0}^{m-1} m_{h}\left(\eta_{j}\right) \int_{x_{i}}^{x_{i+1}}\left[f\left(t, y_{j+1}\right)-f\left(t, y_{j}\right)\right] d t \\
& +\sum_{i=0}^{n-1} \sum_{j=0}^{m-1} l_{j} m_{h}\left(\xi_{i}\right)\left[f\left(x_{i+1}, \eta_{j}\right)-f\left(x_{i}, \eta_{j}\right)\right] \\
& +\sum_{i=0}^{n-1} \sum_{j=0}^{m-1} k_{i} m_{h}\left(\eta_{j}\right)\left[f\left(\xi_{i}, y_{j+1}\right)-f\left(\xi_{i}, y_{j}\right)\right] \\
& -\sum_{i=0}^{n-1} \sum_{j=0}^{m-1} m_{h}\left(\xi_{i}\right) m_{h}\left(\eta_{j}\right)\left[f\left(x_{i}, y_{j}\right)-f\left(x_{i}, y_{j+1}\right)\right. \\
& \left.-f\left(x_{i+1}, y_{j}\right)+f\left(x_{i+1}, y_{j+1}\right)\right]
\end{aligned}
$$

where $k_{i}=x_{i+1}-x_{i}, l_{j}=y_{j+1}-y_{j}(i=0, \ldots, n-1 ; j=0, \ldots, m-1)$, 
$m_{h}\left(\xi_{i}\right)=h\left(\xi_{i}-\frac{x_{i}+x_{i+1}}{2}\right)$ and $m_{h}\left(\eta_{j}\right)=h\left(\eta_{j}-\frac{y_{j}+y_{j+1}}{2}\right)$.

Theorem 7 Let $\mathrm{f}:[\mathrm{a}, \mathrm{b}] \times[\mathrm{c}, \mathrm{d}] \rightarrow \mathbb{R}$ be an absolutely continuous function such that the partial derivative of order 2 exists for all $(\mathrm{t}, \mathrm{s}) \in[\mathrm{a}, \mathrm{b}] \times[\mathrm{c}, \mathrm{d}]$. If $\mathrm{f}_{\mathrm{ts}}=\frac{\partial^{2} \mathrm{f}}{\partial \mathrm{t} \partial \mathrm{s}}$ exists on $(\mathrm{a}, \mathrm{b}) \times(\mathrm{c}, \mathrm{d})$ and is bounded, i.e.,

$$
\left\|f_{t s}\right\|_{\infty}=\sup _{(t, s) \in\left(x_{i}, x_{i+1}\right) \times\left(y_{j}, y_{j+1}\right)}\left|\frac{\partial^{2} f(t, s)}{\partial t \partial s}\right|<\infty .
$$

Then we have the representation

$$
\int_{a}^{b} \int_{c}^{d} f(t, s) d s d t=T\left(f, I_{n}, J_{m}, \xi, \eta\right)+R\left(f, I_{n}, J_{m}, \xi, \eta\right)
$$

where $\mathrm{S}\left(\mathrm{f}, \mathrm{f}^{\prime}, \xi, \mathrm{I}_{\mathrm{n}}\right)$ is defined as in (23) and the remainder satisfies the estimations:

$$
\begin{aligned}
& \left|R\left(f, I_{n}, J_{m}, \xi, \eta\right)\right| \\
& \leq \sum_{i=0}^{n-1} \sum_{j=0}^{m-1}\left[\frac{k_{i}^{2}}{4}+\left(\xi_{i}-\frac{x_{i}+x_{i+1}}{2}\right)^{2}+(h-2)\left(\xi_{i}-\frac{x_{i}+x_{i+1}}{2}\right) m_{h}\left(\xi_{i}\right)\right] \\
& \times\left[\frac{l_{j}^{2}}{4}+\left(\eta_{j}-\frac{y_{j}+y_{j+1}}{2}\right)^{2}+(h-2)\left(\eta_{j}-\frac{y_{j}+y_{j+1}}{2}\right) m_{h}\left(\eta_{j}\right)\right]\left\|f_{t s}\right\|_{\infty}
\end{aligned}
$$

for all $\left(\xi_{i}, \eta_{j}\right) \in\left[x_{i}, x_{i+1}\right] \times\left[y_{j}, y_{j+1}\right]$ with $(i=0, \ldots, n-1 ; j=0, \ldots, m-1)$, where $\mathrm{m}_{\mathrm{h}}\left(\xi_{\mathrm{i}}\right)=\mathrm{h}\left(\xi_{\mathrm{i}}-\frac{x_{i}+x_{i+1}}{2}\right)$ and $\mathrm{m}_{\mathrm{h}}\left(\eta_{j}\right)=\mathrm{h}\left(\eta_{j}-\frac{y_{j}+y_{j+1}}{2}\right)$ with $\mathrm{h} \in$ $[0,2]$.

Proof. Applying Theorem 3 on the interval $\left[x_{i}, x_{i+1}\right] \times\left[y_{j}, y_{j+1}\right],(i=0, \ldots, n-1$; $j=0, \ldots, m-1)$, we obtain

$$
\begin{aligned}
& \mid \int_{x_{i}}^{x_{i+1}} \int_{y_{j}}^{y_{j+1}} f(t, s) d s d t-l_{j} \int_{x_{i}}^{x_{i+1}} f\left(t, \eta_{j}\right) d t-k_{i} \int_{y_{j}}^{y_{j+1}} f\left(\xi_{i}, s\right) d s+k_{i} l_{j} f\left(\xi_{i}, \eta_{j}\right) \\
& \quad+m_{h}\left(\xi_{i}\right) \int_{y_{j}}^{y_{j+1}}\left[f\left(x_{i+1}, s\right)-f\left(x_{i}, s\right)\right] d s+m_{h}\left(\eta_{j}\right) \int_{x_{i}}^{x_{i+1}}\left[f\left(t, y_{j+1}\right)-f\left(t, y_{j}\right)\right] d t \\
& \quad-l_{j} m_{h}\left(\xi_{i}\right)\left[f\left(x_{i+1}, \eta_{j}\right)-f\left(x_{i}, \eta_{j}\right)\right]-k_{i} m_{h}\left(\eta_{j}\right)\left[f\left(\xi_{i}, y_{j+1}\right)-f\left(\xi_{i}, y_{j}\right)\right]
\end{aligned}
$$




$$
\begin{aligned}
& +m_{h}\left(\xi_{i}\right) m_{h}\left(\eta_{j}\right)\left[f\left(x_{i}, y_{j}\right)-f\left(x_{i}, y_{j+1}\right)-f\left(x_{i+1}, y_{j}\right)+f\left(x_{i+1}, y_{j+1}\right)\right] \mid \\
\leq & \left\|f_{t s}\right\|_{\infty}\left[\frac{k_{i}^{2}}{4}+\left(\xi_{i}-\frac{x_{i}+x_{i+1}}{2}\right)^{2}+(h-2)\left(\xi_{i}-\frac{x_{i}+x_{i+1}}{2}\right) m_{h}\left(\xi_{i}\right)\right] \\
& \times\left[\frac{l_{i}^{2}}{4}+\left(\eta_{j}-\frac{y_{j}+y_{j+1}}{2}\right)^{2}+(h-2)\left(\eta_{j}-\frac{y_{j}+y_{j+1}}{2}\right) m_{h}\left(\eta_{j}\right)\right]
\end{aligned}
$$

for all $i=0, \ldots, n-1 ; j=0, \ldots, m-1$.

Summing over $i$ from 0 to $n-1$ and over $j$ from 0 to $m-1$ using the generalized triangle inequality we obtain the estimation (24).

Remark 11 If we take $\mathrm{h}=0$ in Theorem 7 , then we recapture the cubature formula

$$
\int_{a}^{b} \int_{c}^{d} f(t, s) d s d t=T\left(f, I_{n}, J_{m}, \xi, \eta\right)+R\left(f, I_{n}, J_{m}, \xi, \eta\right)
$$

where the remainder $\mathrm{R}\left(\mathrm{f}, \mathrm{I}_{\mathfrak{n}}, \mathrm{J}_{m}, \xi, \eta\right)$ satisfies the estimation:

$$
\begin{aligned}
& \left|R\left(f, I_{n}, J_{m}, \xi, \eta\right)\right| \\
& \leq\left\|f_{t s}\right\|_{\infty} \sum_{i=0}^{n-1} \sum_{j=0}^{m-1}\left[\frac{k_{i}^{2}}{4}+\left(\xi_{i}-\frac{x_{i}+x_{i+1}}{2}\right)^{2}\right]\left[\frac{l_{j}^{2}}{4}+\left(\eta_{j}-\frac{y_{j}+y_{j+1}}{2}\right)^{2}\right]
\end{aligned}
$$

which was given by Barnett and Dragomir in [2].

Remark 12 If we choose $\xi_{i}=\frac{x_{i}+x_{i+1}}{2}$ and $\eta_{j}=\frac{y_{j}+y_{j+1}}{2}$ in Theorem 7 , then we recapture the midpoint cubature formula

$$
\int_{a}^{b} \int_{c}^{d} f(t, s) d s d t=T_{M}\left(f, I_{n}, J_{m}\right)+R_{M}\left(f, I_{n}, J_{m}\right)
$$

where the remainder $R_{M}\left(f, I_{n}, J_{m}\right)$ satisfies the estimation:

$$
\left|R_{M}\left(f, I_{n}, J_{m}\right)\right| \leq \frac{\left\|f_{t s}\right\|_{\infty}}{16} \sum_{i=0}^{n-1} k_{i}^{2} \sum_{j=0}^{m-1} l_{j}^{2} .
$$

Theorem 8 Let $\mathrm{f}:[\mathrm{a}, \mathrm{b}] \times[\mathrm{c}, \mathrm{d}] \rightarrow \mathbb{R}$ be an absolutely continuous function such that the partial derivative of order 2 exists for all $(\mathrm{t}, \mathrm{s}) \in[\mathrm{a}, \mathrm{b}] \times[\mathrm{c}, \mathrm{d}]$. If 
$\left|\mathrm{f}_{\mathrm{ts}}(\mathrm{t}, \mathrm{s})\right|^{\mathrm{q}}$ is a convex function on the co-ordinates on $[\mathrm{a}, \mathrm{b}] \times[\mathrm{c}, \mathrm{d}], \frac{1}{\mathrm{p}}+\frac{1}{\mathrm{q}}=1$ and $\mathrm{q}>1$, then we have the representation

$$
\int_{a}^{b} \int_{c}^{d} f(t, s) d s d t=T\left(f, I_{n}, J_{m}, \xi, \eta\right)+R\left(f, I_{n}, J_{m}, \xi, \eta\right)
$$

where $\mathbf{S}\left(f, f^{\prime}, \xi, I_{n}\right)$ is defined as in (23) and the remainder satisfies the estimations:

$$
\begin{aligned}
& \left|R\left(f, I_{n}, J_{m}, \xi, \eta\right)\right| \\
& \leq \sum_{i=0}^{n-1} \sum_{j=0}^{m-1} k_{i}^{\frac{1}{q}} l_{j}^{\frac{1}{q}}\left[\frac{\left[x_{i+1}-\xi_{i}+m_{h}\left(\xi_{i}\right)\right]^{p+1}+\left[\xi_{i}-x_{i}-m_{h}\left(\xi_{i}\right)\right]^{p+1}}{p+1}\right]^{\frac{1}{p}} \\
& \quad \times\left[\frac{\left[y_{j+1}-\eta_{j}+m_{h}\left(\eta_{j}\right)\right]^{p+1}+\left[\eta_{j}-y_{j}-m_{h}\left(\eta_{j}\right)\right]^{p+1}}{p+1}\right]^{\frac{1}{p}} \\
& \quad \times\left\{\frac{\left|f_{t s}\left(x_{i}, y_{j}\right)\right|^{q}+\left|f_{t s}\left(x_{i}, y_{j+1}\right)\right|^{q}+\left|f_{t s}\left(x_{i+1}, y_{j}\right)\right|^{q}+\left|f_{t s}\left(x_{i+1}, y_{j+1}\right)\right|^{q}}{4}\right\}^{\frac{1}{q}}
\end{aligned}
$$

for all $\left(\xi_{i}, \eta_{j}\right) \in\left[x_{i}, x_{i+1}\right] \times\left[y_{j}, y_{j+1}\right]$ with $(i=0, \ldots, n-1 ; j=0, \ldots, m-1)$, where $\mathrm{m}_{\mathrm{h}}\left(\xi_{i}\right)=\mathrm{h}\left(\xi_{i}-\frac{x_{i}+x_{i+1}}{2}\right)$ and $\mathrm{m}_{\mathrm{h}}\left(\eta_{j}\right)=\mathrm{h}\left(\eta_{j}-\frac{y_{j}+y_{j+1}}{2}\right)$ with $\mathrm{h} \in$ $[0,2]$.

Proof. Applying similar methods in the proof of Theorem 7 and then using the inequality (21), we obtain desired result.

\section{References}

[1] F. Ahmad, N. S. Barnett and S. S. Dragomir, New Weighted Ostrowski and Cebysev Type Inequalities, Nonlinear Analysis: Theory, Methods \& Appl., 71 (12) (2009), 1408-1412.

[2] N. S. Barnett and S. S. Dragomir, An Ostrowski type inequality for double integrals and applications for cubature formulae, Soochow J. Math., 27 (1) (2001), 109-114.

[3] M. K. Bakula, M. E. Özdemir and J. Pečarić, Hadamard type inequalities for $\mathfrak{m}$-convex and $(\alpha, \mathfrak{m})$-convex functions, J. Inequal. Pure and Appl. Math. 9 (4) (2008), Art. 96. 
[4] F. Chen, On Hermite-Hadamard type inequalities for s-convex functions on the coordinates via Riemann-Liouville fractional integrals, J. of Appl. Math., Volume 2014, Article ID 248710, 8 pages.

[5] S. S. Dragomir, On Hadamard's inequality for convex functions on the coordinates in a rectangle from the plane, Taiwanese J. of Math., 4 (2001), $775-788$.

[6] S. S. Dragomir, N. S. Barnett and P. Cerone, An Ostrowski type inequality for double integrals in term of $\mathrm{L}_{\mathrm{p}}-$ norms and Applications in numerical integrations, Anal. Num. Theor. Approx., 2 (12) (1998), 1-10.

[7] S. Erden and M. Z. Sarikaya, On the Hermite- Hadamard's and Ostrowski's inequalities for the co-ordinated convex functions, RGMIA Research Report Collection, Vol. 18 (2015). Art. 57.

[8] S. Erden and M. Z. Sarikaya, On the Hermite-Hadamard-Type and Ostrowski Type inequalities for the co-ordinated convex functions, Palestine J. of Math., in press.

[9] J. Hadamard, Etude sur les proprietes des fonctions entieres et en particulier d'une fonction considree par, Riemann, J. Math. Pures. et Appl. 58 (1893), 171-215.

[10] M. A. Latif and M. Alomari, Hadamard-type inequalities for product two convex functions on the co-ordinetes, Int. Math. Forum, 4 (47) (2009), $2327-2338$.

[11] M. A. Latif and M. Alomari, On the Hadamard-type inequalities for hconvex functions on the co-ordinetes, Int. J. of Math. Analysis, 3 (33) (2009) 1645-1656.

[12] M. A. Latif and S.S. Dragomir, On some new inequalities for differentiable co-ordinated convex functions, J. of Inequalities and Appl., (2012), 2012:28.

[13] M. A. Latif, S. Hussain and S. S. Dragomir, New Ostrowski type inequalities for co-ordinated convex functions, TJMM, 4 (2) (2012), 125-136.

[14] M. E. Ozdemir, E. Set and M.Z. Sarikaya, New some Hadamard's type inequalities for coordinated $\mathrm{m}$-convex and $(\alpha, \mathrm{m})^{\mathrm{n}}$-convex functions, Hacettepe J. of Math. and Statistics, 40 (2) (2011), 219-229. 
[15] A. M. Ostrowski, "Uber die absolutabweichung einer differentiebaren funktion von ihrem integralmitelwert, Comment. Math. Helv. 10 (1938), $226-227$.

[16] B. G. Pachpatte, On a new Ostrowski type inequality in two independent variables, Tamkang J. Math., 32 (1) (2001), 45-49.

[17] J. Park, Some Hadamard's type inequalities for co-ordinated (s;m)convex mappings in the second sense, Far East J. of Mathematical Sciences, 51 (2) (2011), 205-216.

[18] A. Rafiq and F. Ahmad, Another weighted Ostrowski-Grüss type inequality for twice differentiable mappings, Kragujevac J. of Math., 31 (2008), $43-51$.

[19] M. Z. Sarikaya and H. Ogunmez, On the weighted Ostrowski type integral inequality for double integrals, The Arabian J. for Science and Eng. (AJSE)-Math., (2011) 36: 1153-1160.

[20] M. Z. Sarikaya, On the Ostrowski type integral inequality, Acta Math. Univ. Comenianae, Vol. LXXIX, 1 (2010), 129-134.

[21] M. Z. Sarikaya, E. Set, M. E. Özdemir and S. S. Dragomir, New some Hadamard's type inequalities for co-ordineted convex functions, Tamsui Oxford J. of Information and Mathematical Sciences, 28 (2) (2012), 137152.

[22] M. Z. Sarikaya, On the Hermite-Hadamard-type inequalities for coordinated convex function via fractional integrals, Integral Transforms and Special Functions, Vol. 25, No. 2 (2014), 134-147.

[23] M. Z. Sarikaya, Some inequalities for differentiable co-ordinated convex mappings, Asian-European J. of Math., Vol: 08, 1550058 (2015), 21 pages.

[24] M. Z. Sarikaya, On the generalized weighted integral inequality for double integrals, Annals of the Alexandru Ioan Cuza University - Math., 61 (1) (2015), 169-179. 